\title{
Astroturismo: uma análise no Parque Estadual Morro do Diabo, Teodoro Sampaio, São Paulo
}

\section{Astrotourism: an analysis at "Morro do Diabo" State Park, in Teodoro Sampaio, São Paulo, Brazil}

\author{
Vitor Barbato Honorato ${ }^{1}$ \\ Fabio Luciano Violin ${ }^{2}$
}

\begin{abstract}
Resumo: $\mathrm{O}$ crescimento do segmento de turismo ligado à natureza nos últimos anos possibilita a abertura de novos mercados com o mesmo intuito. $\mathrm{O}$ astroturismo terrestre é um modelo de nicho que pode se aproveitar desse aumento de interesse tanto turístico quanto em relação à astronomia. $\mathrm{O}$ estudo trata das principais bases do astroturismo, estudando o que delimita suas implicações e benefícios, considerando posteriormente o Parque Estadual Morro do Diabo (PEMD), localizado no extremo oeste do estado de São Paulo, pertencente ao município de Teodoro Sampaio, como exemplo de uma possível aplicação. A pesquisa baseia-se na proposta do astroturismo como uma alternativa de turismo sustentável e na sua possibilidade de implantação analisando um local. Demonstrou-se que este segmento turístico é benéfico em diversas áreas, tanto em relação a saúde humana, qualidade ambiental e proteção de patrimônio mundial, devendo ser aplicado em áreas que têm possibilidade de acomodação desse tipo de atividade, elemento passível de consideração no local analisado.
\end{abstract}

Palavras-chave: Turismo; Astroturismo; Turismo Sustentável; Parque Estadual.

Abstract: The growth of the nature-related tourism segment in recent years has made it possible to open new markets for the same purpose. The terrestrial astrotourism is a niche model that can take advantage of this increased interest in both tourism and astronomy. The study deals with the main bases of astrotourism, investigation what delimits its implications and benefits, considering then the Morro do Diabo State Park (PEMD), located in the extreme west of the São Paulo State, belonging to the municipality of Teodoro Sampaio, as an example of a possible application. The research is based on the propose of astrotourism being a sustainable tourism alternative and in its possibility of implantation by analyzing a place. It has been shown that this tourism

\footnotetext{
${ }^{1}$ Graduação em Turismo pela Universidade Estadual Paulista Júlio de Mesquita Filho (UNESP).

E-mail: vitor.barbato@outlook.com

${ }^{2}$ Doutorado em Meio Ambiente e Desenvolvimento Regional pela Universidade Anhanguera Uniderp. Professor da Universidade Estadual Paulista Júlio de Mesquita Filho (UNESP). E-mail: fabio.violin@unesp.br
} 
segment is beneficial in several areas, such as human health, environmental quality and world heritage protection, and it should be applied in areas that can accommodate this type of activity, an element that may be considered in the analyzed place.

Keywords: Tourism; Astrotourism; Sustainable Tourism; State Park. 


\section{INTRODUÇÃO}

Desde os primórdios da humanidade (MARIN, 2010) o homem já se questionava sobre os diversos eventos que aconteciam no céu, inclusive, conseguia conjecturar possíveis viagens para fora do que o cercava na Terra. O céu tem sua importância para a história da humanidade, utilizando-o como referência na agricultura, caça, pesca e navegações marítimas, a exemplo das realizadas no século XIV e XV, além de outras atividades cotidianas (TOMANIK; BASTOS, 2012).

Assim, o céu noturno proporciona uma experiência ao observador, podendo mudar perspectivas, fomentar inspirações, e direcionar para refletir sobre a humanidade e seu lugar no universo. Dentre essas possibilidades está o astroturismo, que tem como seu principal recurso o céu. Nele encontra-se toda natureza do universo, e os astros nele, por enquanto, intangíveis para o homem. Esse segmento traz consigo a preocupação com a poluição luminosa e o turismo sustentável. A poluição luminosa é produto da sociedade industrial, e sua fonte provém de diversos estabelecimentos, residências, fábricas, iluminação de vias públicas, outdoors, entre outros, e muitas vezes essas luzes seriam desnecessárias, ou estão mal adaptadas (IDA, 2019a).

Além de ser uma atividade com reduzido impacto, ela também pode tem a potencialidade na implantação do astroturismo em locais que já fornecem estrutura apresenta-se como de baixo custo relativo. Assim, pode compor um produto turístico isolado ou mesmo casado com outras ofertas no turismo.Corroborando com tal apontamento, Chibiaqui e Neto (2018) destacam que "o turismo é uma importante atividade sob os pontos de vista econômico e do desenvolvimento sustentável.

Assim, esse estudo objetiva um esclarecimento a respeito do astroturismo, identificando a sua implantação no Parque Estadual Morro do Diabo, como exemplo de possibilidade de sua aplicação. Para tanto inicia com uma revisão dos conceitos de poluição luminosa, seus benefícios quando evitada, e algumas instituições que cuidam de tal fator. Em seguida, é apresentado o astroturismo, relacionando-o ao turismo sustentável devido aos componentes presentes em sua aplicação. Então são apresentados os resultados e as considerações finais. 


\section{REFERENCIAL TEÓRICO}

\subsection{DARK-SKY E POLUIÇÃO LUMINOSA}

A crescente preocupação com o meio ambiente nas últimas décadas desencadeou uma série de pesquisas sobre impactos da poluição do solo, das águas e do ar (RODRIGUES et al., 2014). Uma área não muito analisada, principalmente no turismo, é a da poluição luminosa. Segundo a International Dark-Sky Association (IDA, 2019b), a poluição luminosa se caracteriza pelo uso inapropriado, ou excessivo de luz artificial, sendo que o brilho exacerbado causado pela iluminação gera uma série de fatores como: desconforto nos olhos, iluminação em céus sobre áreas não habitadas, luzes invasivas onde não deveriam chegar, e confusão das fontes de luz quando em maior quantidade de emissores. Assim, a poluição luminosa pode ser dividida entre a luz que invade algum local natural ou que teria pouca luz, e a que tem uso de luz excessiva, que normalmente é ligado ao desconforto e gera problemas de saúde (RODRIGUES et al., 2014).

Os malefícios não remetem somente a viabilidade de observação do céu noturno, este tipo de poluição, presente desde o século XIX é prejudicial para o ciclo de vida dos animais devido a implantação dos primeiros tipos de iluminação artificial (RICH; LONGCORE, 2013). Por exemplo, o quadro de Van Gogh denominado de "A Noite Estrelada", pintado em Saint Rémy, na França, no ano de 1889, nos dias de hoje o pintor não conseguiria ver a Via Láctea, que inspirou o quadro (IDA, 2019c).

Uma pesquisa realizada pelo World Atlas of Artificial Night Sky Brightness demonstra que $83 \%$ da população mundial, e $99 \%$ dos europeus e moradores dos Estados Unidos, vivem sob um céu que não tem brilho natural, pelo contrário, tem pelo menos $10 \%$ de alteração de iluminação artificial (FALCHI et al., 2016). Por volta de $14 \%$ de população mundial nem consegue usar a visão acostumada para um ambiente noturno, pois a noite está tão clara que as configurações do olhar são iguais a de como se estivesse de dia quando olhando para o céu. Isto demonstra uma necessidade para a preocupação com a poluição luminosa, já que $1 / 3$ da população mundial não consegue ver a Via Láctea por tais motivos (MORELLE, 2016). Até nos animais ocorrem efeitos desta poluição em seus ciclos de alimentação, comunicação e reprodução das diversas espécies (LONGCORE; RICH, 2004; IDA, 2019d). 
Frente a esse cenário e à sua importância, o céu foi considerado patrimônio da humanidade pela sua característica natural e cultural. E o ano de 2009 foi reconhecido pela UNESCO, como o Ano Internacional da Astronomia de 2009. A sua relevância é evidenciada a seguir que:

O céu, nossa herança universal e em comum, é parte integral do ambiente percebido pela natureza humana. A humanidade sempre observou o céu tanto para observar, quanto para entender as leis da física que governam o universo. Este interesse na astronomia fez profundas implicações na ciência, filosofia, religião, cultura e nossa concepção geral do universo (UNESCO, 2009, p.1).

Algumas instituições foram criadas com intuitos de preservação e proteção desse patrimônio. Entre elas, estão a International Dark-Sky Association (IDA). Fundada em 1988, tem como objetivos educar a público e os políticos sobre a conservação do céu noturno, batalhar pela proteção de tal, promover uma iluminação ambientalmente responsável, além de ajudar o público com recursos para a restauração da noite como era antes (IDA, 2019e).

[...] o conceito Dark-Sky visa sensibilizar, por via da criação de uma rede de destinos, o respeito por um conjunto de boas práticas, direcionadas a uma política de consumo energético responsável, a uma preocupação com os impactos no meio ambiente, na saúde e centrada na ideia de que a observação do céu é um direito de todos (DICK; WELCH, 2012, p.37).

No intuito de proteger e exercer a preservação do céu noturno a IDA tem um programa que cria reservas de dark-sky, sendo estas, reconhecidas por promover a administração responsável do céu noturno. Já há mais de 65 lugares pelo mundo protegidos pela associação, distribuídos nos 6 continentes, entretanto a maioria se concentra nos Estados Unidos e no Canadá, no total correspondem a mais de 58 mil quilómetros quadrados de extensão com esse tipo de proteção (IDA, 2019b). A associação já publicou diversos artigos, guias e jornais com destaque para a poluição luminosa, além de organizar eventos, encontros, e programas com o público, criando selos de aprovação, comunidades internacionais, parques e reservas de dark-sky (RODRIGUES et al., 2014). Todas essas medidas asseguram que não só a presente, mas as futuras gerações, tenham também a possibilidade da visualização do patrimônio mais universal e compartilhado entre todos os povos, o céu noturno. 
Além da IDA, iniciativas como a International Initiative in Defense of the Quality of the Night Sky e a Mankind's Scientific, Cultural and Environmental Right (Starlight Initiative) promovem não só a preservação do céu noturno, mas também, sua proteção enquanto patrimônio. A Starlight Foundation é responsável pela gestão operacional da iniciativa (RODRIGUES et al., 2014). Fundada em 2007, já conta com a certificação de 17 destinos com o selo de Starlight Tourism Destination, para isso, tem o apoio de órgãos como a Organização das Nações Unidas (ONU), Organização das Nações Unidas para a Educação, a Ciência e a Cultura (UNESCO) e a Organização Mundial do Turismo (OMT) (MAIA, 2017). A preocupação com visibilidade do céu noturno é essencial para a prática do astroturismo, analisado a seguir.

\subsection{ASTROTURISMO: CARACTERÍSTICAS E SUA RELAÇÃO COM O TURISMO SUSTENTÁVEL}

A predisposição de viajar com o motivo da observação de eventos astronômicos e a área de astronomia vem crescendo nos últimos tempos. Diversos fatores têm possibilitado a essa área um acesso mais voltado ao público geral; dentre estas a atuação de cientistas como Carl Sagan e Neil DeGrasse Tyson, disseminadores científicos que apelaram a esse modelo voltado a um público maior.

O turismo astronômico ou astroturismo, simplificadamente, existe e se conecta há várias centenas de anos pelo mundo. Este fenômeno tem como destinos, por exemplo, Nabta Playa no Deserto do Sahara, o Stonehenge no Reino Unido, Machu Pichu no Peru e as Pirâmides de Gizé no Egito. Segundo Malville (2008), no passado a observação das estrelas para era utilizada para localização por parte dos seres humanos tinha propósitos específicos relacionados à agricultura ou outras práticas culturais e tradicionais. Hoje essa prática é utilizada por turistas, porém com um propósito diferente, como a astrofotografía.

Atualmente, o astroturismo se desenvolveu e inclui atividades de simples observação de estrelas, viagens para lugares específicos para observação de auroras ou eclipses, até a visitação de centros de comando espaciais ou estações espaciais (MATOS, 2017). A observação do céu pode contar com diversos tipos de motivos, incluindo fenômenos pontuais, como eclipses, chuvas de meteoros, aparição de planetas 
e cometas. Há também a motivação ligada a quantidade de poluição luminosa e atmosférica que uma área consegue se restringir, possibilitando uma maior visualização de todos os tipos de astros presentes no céu daquela região que normalmente as cidades escondem (MAIA, 2017). Para a população que mora em locais com poluição luminosa, o céu noturno percebido de suas casas é o respectivo de algumas estrelas mais brilhantes, assim como planetas e um brilho exercido pela iluminação artificial (COLLISON; POE, 2013).

A maior parte das pessoas que procura o astroturismo é composta por cientistas, pesquisadores e astrônomos amadores. Entretanto, segundo Gibbs e Beredsen (2007) astrônomos amadores podem ser tão efetivos quanto astrônomos profissionais na divulgação científica. Astrônomos amadores com mais conhecimento são mais prováveis de fazer essa divulgação, especialmente se ligados a clubes de astronomia (COLLISON; POE, 2013).

Desse modo o astroturismo também traria resultados para o campo científico, se divulgado e promovido como uma área de proteção a um dos instrumentos de trabalho da astronomia, o céu noturno. Além disso, proporciona uma experiência diferente ao turista comum. Segundo Krippendorf (2000), o turismo é uma possibilidade de escapar da rotina do dia a dia deste modo, fazendo o indivíduo mais feliz. O astroturismo incorpora a recente tendência de ter uma experiência mais significativa, principalmente as focadas na conservação dos recursos naturais (céu noturno), conhecimento e ciência, convidando não somente o turista individual mas grupos de família também (SOLÁ et al., 2014).

Um dos exemplos desse tipo de aplicação de turismo foi a criação da Reserva Dark Sky de Alqueva, em Portugal, que teve o propósito de proteger o céu noturno da região e promover o turismo. A reserva reúne seis cidades na região do Alantejo em Portugal, combinando turismo rural com stargazing (observação de estrelas). Devido a isso, atrai diversos turistas, pois além da possibilidade de observação, o local possui passeios de barco, cavalgadas e caminhadas noturnas, bem como aulas de fotografia e astronomia (MAIA, 2017).

$\mathrm{O}$ astroturismo ainda se divide em astroturismo terrestre, que por sua vez pode incluir visitas a observatórios, centros espaciais, satélites e parques de telescópios, parques de Dark Sky entre outros. Neste artigo, será tratado sobre o astroturismo 
terrestre, focado na área de reservas e parques, devido a características do Parque Estadual Morro do Diabo (PEMD) (MATOS, 2017).

Em termos conceituais, há diferentes definições. Spennemann (2008) coloca que o astroturismo consiste em viagens de indivíduos para localizações específicas para satisfazer o desejo de ver planetas e estrelas, sendo a olho nu, ou com algum tipo de dispositivo óptico como telescópios. Collison e Poe (2013) definem que é um segmento do turismo sustentável (como sua fonte é o céu no período da noite, que não é empobrecido ou sofre danos permanentemente por ser observado) que envolve participação do público em visualização de objetos astronômicos em áreas com ausência de poluição da iluminação artificial. Solá et al. (2014) definem o astroturismo como atividade de viajantes que desejam usar o recurso natural de locais bem preservados de paisagens noturnas para lazer e conhecimento relacionado a astronomia. A prática do astroturismo enquanto uma atividade sustentável será abordada a seguir.

O astroturismo se relaciona turismo sustentável primeiramente, pois como expõe Marin (2010) “o turismo pode se tornar um instrumento de aliança na defesa da qualidade da paisagem noturna. O turismo responsável, em muitas formas, pode e deve tomar o céu noturno como um recurso a proteger e valorizar em todas as destinações".

A geração de novos produtos turísticos baseados na observação do céu e dos fenômenos da noite, também abre possibilidades para a cooperação entre a comunidade local, instituições científicas e partes interessadas no tipo de turismo (RODRIGUES et al., 2014). Além disso, há a possibilidade do aumento da conscientização das organizações e da comunidade com a poluição luminosa, e assim possíveis mudanças nos modelos de iluminação do sistema da cidade (RODRIGUES et al., 2014).

Assim, o astroturismo possibilita uma forma de lazer, tem caráter educativo e se configura como "estratégia de revitalização do tecido social e econômico de uma dada região" (TOMANIK; BASTOS, 2012, p. 386). Portanto, ele se caracteriza como uma medida sustentável, que além da parte ambientalmente responsável, ainda pode gerar renda e ajudar no equilíbrio de uma cidade que tenha proximidade com a natureza com seu fluxo turístico.

Najafabadi (2012, p.130) defendem que "existindo potencial para o astroturismo num determinado destino, este, deve ser expandido e desenvolvido". Por isso, a seguir, a 
seguir serão apresentados os procedimentos metodológicos e os resultados da pesquisa sobre a sua possível implantação no Parque Estadual Morro do Diabo.

\section{METODOLOGIA}

O estudo tem no aspecto qualitativo sua natureza primeira e dentro do contexto estruturante da pesquisa, o estudo de caso apresenta-se como o elemento central direcionador das decisões metodológicas. Nesses termos Lüdke e André (1986, p.257) apontam que "o caso é sempre bem delimitado, devendo ter seus contornos claramente definidos no desenvolver do estudo". Considerando as pretensões já apontadas, o estudo de caso é o mais apropriado pois a situação a qual se refere o conjunto de dados permite o aprofundamento na situação particular e singular que vai desde a coleta até a análise dos insumos informacionais.

Moreira (2002, p. 52), indica que a observação participante representa "uma estratégia de campo que combina ao mesmo tempo a participação ativa com os sujeitos, a observação intensiva em ambientes naturais, entrevistas abertas informais e análise documental". Nesse caso específico, a observação participante instrumentaliza-se a partir da observação in loco dentro do ambiente do Parque Estadual Morro do Diabo, no sentido de levantar os aspectos de acesso, estrutura, funcionamento e pertinência considerando a proposta envolvendo os preceitos do Astroturismo.

A pesquisa possui contornos descritivos e explicativos no conjunto de suas interpretações e transposição da situação de dados para informações ao longo da estruturação e contextualização do estudo. O caráter descrito apresenta-se como inerente ao contexto proposto pois, segundo Vergara (2000, p.47) esse tipo de pesquisa "não têm o compromisso de explicar os fenômenos que descreve, embora sirva de base para tal explicação", porém estabelece as necessárias correlações entre variáveis além de definir sua natureza.

A coleta de dados se deu por meio de análise documental em sites especializados e artigos específicos além da observação in loco e registro fotográfico no local de análise. A análise dos dados ocorreu a partir do conjunto de dados levantados e buscou aferir a viabilidade da proposta ou elementos que a refutassem. 


\section{O PARQue ESTAdUAL MORRO DO DIABO E A POSSIBILIDADE DE ASTROTURISMO}

O Parque Estadual Morro do Diabo PEMD situa-se em Teodoro Sampaio, município da região do Pontal do Paranapanema. Os turistas que visitam o parque procuram turismo ecológico, o ecoturismo, o turismo rural e o turismo de natureza focado na pesca. O Parque foi criado em 1941 como uma reserva e tornou-se parque em 1986, sendo considerado patrimônio de biodiversidade. A área abriga uma das últimas reservas de floresta de planalto do país e é uma das áreas-núcleo da Reserva da Biosfera da Mata Atlântica Brasileira (SÃO PAULO, 2017). O parque preserva a maior área contínua remanescente da floresta que recobria a porção ocidental do estado de São Paulo e de estados vizinhos, que se refere à mais de 33 mil hectares de extensão, área que remanesce diversas espécies de fauna e flora, contendo vegetação não só de Mata Atlântica, como também, há características da flora do cerrado (SÃO PAULO, 2010).

A respeito dos mamíferos, encontram-se no Parque algumas espécies endêmicas ao bioma da Mata Atlântica, bem como espécies consideradas em risco de extinção por organismos nacionais e internacionais. Tomando em conta apenas espécies cuja ocorrência foi recentemente confirmada, há um total de 4 espécies endêmicas, sete espécies presentes na Lista Oficial Brasileira da Fauna Ameaçada de Extinção e 17 espécies presentes na Lista Oficial da Fauna Ameaçada de Extinção do Estado de São Paulo (FARIA et al., 2012).

A partir das informações advindas da Light Pollution Map (2019) tem-se que a área em que o pico do morro é estabelecido, tem média de poluição luminosa de 0.17 , em escala de 8 componentes no sentido crescente de poluição luminosa. Nessa escala o primeiro é menor que 0.25 , caso em que o morro se encontra. O segundo componente é de 0.25 a 0.40 , o terceiro de 0.40 a 1.00 , o quarto de 1.00 a 3.00 , o quinto de 3.00 a 6.00 , o sexto de 6.00 a 20.00, o sétimo de 20.00 a 40.00 e o oitavo de 40.00 para cima. A maior parte do de São Paulo centro da capital se encontra nesse último caso, com médias de 70.00. A medida é baseada em dados fornecidos por satélite VIIRS com responsabilidade dos dados de Earth Observation Group, NOAA National Geophysical Data Center. O resultado do índice de poluição luminosa no PEMD é comparável e ao 
da região de Alantejo. Além disso, a elevação do morro traz vantagens em relação a observação mais próxima ao nível do mar (LIGHT POLLUTION MAP, 2019).

O PEMD já realiza visitas agendadas no período da manhã no pico do morro, entretanto não há ainda a possibilidade de visitações turísticas noturnas. Em relação a estruturação da trilha do Morro do Diabo, há no local, placas de identificação, que orientam e informam o visitante sobre diversos assuntos como a diversidade de fauna do morro, corrimão presente na maior parte da subida, bancos no meio e no final do percurso. No cume há estruturas de observação como o mirante com aproximadamente 8 metros de comprimento e 6 metros de largura, que proporciona uma melhor vista da paisagem do alto do morro.

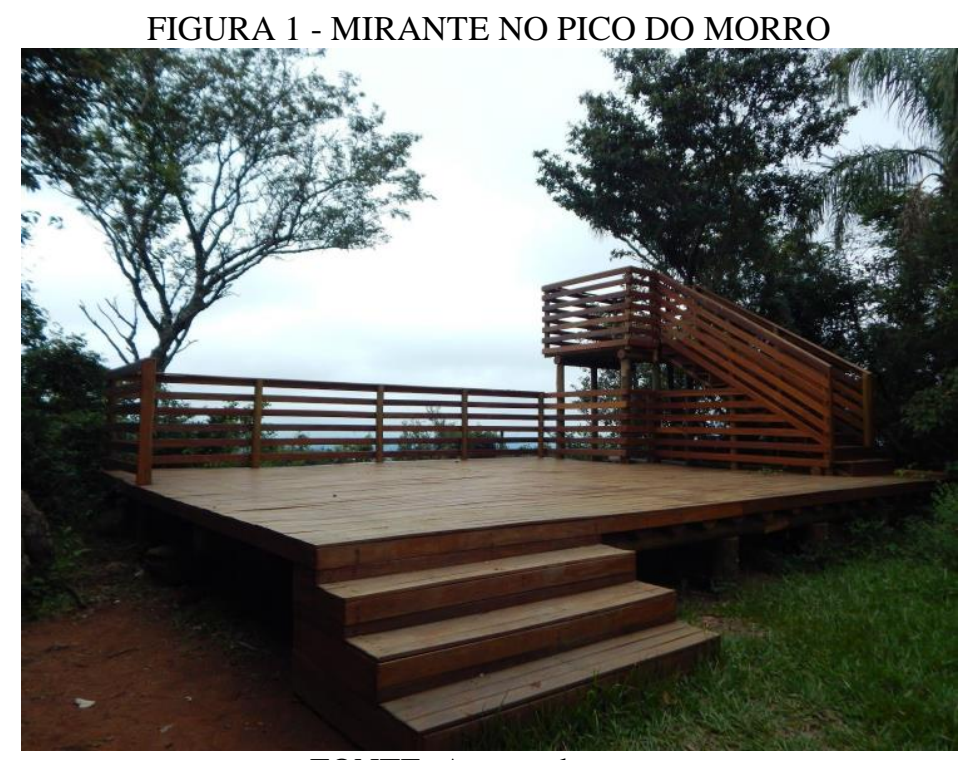

FONTE: Acervo do autor

No mirante ainda há um segmento da estrutura que segue para uma área 2 metros mais elevada, porém mais estreita, por volta de 2 metros de comprimento e de largura, entretanto ambas poderiam servir como recurso na prática do astroturismo.

Para a implantação do astroturismo no Parque é importante a oferta desse produtor em agências de viagem, meios de hospedagem locais, setor de alimentos e bebidas. Ainda seriam necessárias a estrutura de deslocamento, a oferta de produtos artesanais e a organização de eventos locais. Outras organizações ao longo do trecho de deslocamento também poderiam trabalhar com o público da atividade, além da extensão da visitação para outras atividades da localidade como a pesca, turismo rural e similares. Para tanto é importante, como exposto por Gomes (2010) a integração entre os agentes 
os locais envolvidos na cadeia do produto. Dessa forma, o astroturismo poderia estimular o desenvolvimento sustentável da região gerando benefícios de ordem social e valorização da comunidade rural do entorno, que atuará além do comércio de produtos locais e ainda

\section{CONCLUSÃO}

O estudo almejou tratar das principais bases do astroturismo contextualizando sua potencialidade de oferta futura baseada no PEMD discutindo potenciais implicações e benefícios da atividade na localidade. Foi possível identificar que o astroturismo terrestre é um segmento turístico de nicho, com potencialidade de se desenvolver em áreas onde o turismo ainda não é desenvolvido, mas também em locais com presença da atividade, isto devido a sua flexibilidade de adaptação e facilidade de aplicação do modelo.

O segmento pode trazer benefícios para o local que o recebe desenvolvendo a economia e promovendo a preservação de um patrimônio universal: o céu noturno. No Brasil, há poucos lugares que desenvolvem este tipo de segmento. Entretanto, sua integração no plano turístico de uma região, tem potencial para ampliar a quantidade de turistas.

O PEMD tem condições de desenvolver o astroturismo terrestre, pois é localizado em área com uma quantidade de poluição luminosa mínima, tem locais altos para observação, como o pico do morro, além de uma estrutura já montada. Ele também possui sistema de visitas turísticas guiadas, porém somente durante o período da manhã. Dessa forma, sua aplicação traria melhora na qualidade de vida dos habitantes da região a partir da capacidade de gerar renda para os autóctones tanto direta quanto indiretamente.

Assim, o astroturismo traz a possibilidade de ampliação de atrativos em locais que já ofertam o turismo, bastando para tanto a forma de estruturação correta dos equipamentos, materiais, localidade, aliados a capacidade de propagação desse tipo de oferta ao mercado. Deve ser considerada a sua novidade no Brasil, o que torna sua lógica de direcionamento aos potenciais consumidores algo interessante do ponto de 
vista de aproveitamento de estrutura em parques, unidades habitacionais e tantas outras possibilidades dentro do campo do turismo.

A pesquisa apresentada traz resultados no campo prático ao demonstrar um segmento pouco desenvolvido do turismo no Brasil, com o intuito de não só desenvolver as economias locais, mas também em termos de poluição luminosa. Além disso, o astroturismo terrestre promove conjuntamente com políticas de preservação do céu noturno, sua continuação como patrimônio, protegendo para as futuras gerações a oportunidade de estar em contato com o céu noturno.

Do ponto de vista das limitações do estudo, indica-se a ausência de organizações que trabalhem o astroturismo no país, o que dificulta a análise comparativa em termos de potencialidade. Ocorreu também a dificuldade de acesso a dados e informações pertinentes a forma de estruturação desse tipo de oferta tanto no Brasil quanto em localidades de efetiva implantação, o que torna o conjunto de percepções relativamente abstratos. Assim, sugere-se que pesquisas futuras realizem mapeamento dos potenciais locais de implementação desse tipo de oferta, ampliando a análise inclusive dos benefícios nos campos da sustentabilidade e da mercadologia.

\section{REFERÊNCIAS}

CHIBIAQUI, R.; HACK NETO, E. Customer-Based Brand Equity do Complexo Turístico Itaipu: uma comparação entre as percepções dos visitantes do lado brasileiro e paraguaio. Turismo e Sociedade. v. 11, n. 3, p. 495-517, set./dez. de 2018.

COLLISON, F. M.; POE, K. Astronomical Tourism: The Astronomy and Dark Sky Program at Bryce Canyon National Park. Tourism Management Perspectives, v. 7, p.1-15, jul. 2013.

DICK, R.; WELCH, D. International Dark-sky Efforts. In: Environmental Impact of Light Pollution and Its Abatement: Special Report Journal of the Royal Astronomical Society of Canada, p. 22-25, 2012.

FALCHI, F. CINZANO, P.; DURISCOE, D.M.; KYBA, C.C.M. The new world atlas of artificial night sky brightness. Science Advances, v. 2, n. 6, p.1-25, jun. 2016.

FARIA, H. H. (Org.). Parque Estadual Morro do Diabo: Plano de Manejo. Santa Cruz do Rio Pardo: Editora Viena, 2012. 312 p. Disponível em: <http://arquivos.ambiente.sp.gov.br/fundacaoflorestal/2012/01/morrododiabo.pdf>. Acesso em: 24 nov. 2018.

GIBBS, M. G.; BERENDSEN, M. Effectiveness of amateur astronomers as informal science educators. Astronomy Education Review, v. 5, n. 2, p. 114-126, 2007. 
GOMES, B.M.A. Redes organizacionais e canais de distribuição no turismo Turismo e Sociedade, v.3, n.1, p. 37-50, abr. 2010.

INTERNATIONAL DARK-SKY ASSOCIATION (IDA). Light Pollution. 2019a. Disponível em: <https://www.darksky.org/light-pollution/>. Acesso em: 16 abr. 2019.

Light Pollution Effects

on Wildlife and Ecosystems. 2019b. Disponível em: <https://www.darksky.org/lightpollution/wildlife/>. Acesso em: 16 abr. 2019.

2019 c. Disponível em: <http://www.darksky.org/light-pollution/night-skyNight Sky Heritage. heritage/>. Acesso em: 23 abr. 2019.

About. 2019d.

Disponível em: <https://www.darksky.org/about/>. Acesso em: 23 abr. 2019.

Our Work. 2019e.

Disponível em: <https://www.darksky.org/our-work/ >. Acesso em: 23 abr. 2019.

KRIPPENDORF, J. Sociologia do Turismo: para uma nova compreensão do lazer e das viagens. São Paulo: Editora Aleph, 2000.

LIGHT POLLUTION MAP. Radiance information. 2019. Disponível em: <https:// www.lightpollutionmap.info/\#zoom=12\&lat=-2573887\&lon=-5822655\&layers=B0TFF FFFFFFF>. Acesso em: 02 mai. 2019.

LONGCORE, T.; RICH, C. Ecological light pollution. Frontiers in Ecology and the Environment, v. 2, n.4, p. 191-198, 2004.

LÜDKE, M.; ANDRÉ, M. E. D. A. Pesquisa em educação: abordagens qualitativas. São Paulo: EPU, 1986.

MAIA, E. Astroturismo: os melhores lugares para observar estrelas no mundo. 2017. Disponível em: <https://oglobo.globo.com/boa-viagem/astroturismo-os-melhoreslugares-para-observar-estrel as-no-mundo-20895283 >. Acesso em: 27 abr. 2019.

MALVILLE, J. A guide to prehistoric astronomy in the southwest. Johnson Books, 2008.

MARIN, C.; WAINSCOAT, R.; FAYOS -SOLÀ, E. Windows to the Universe: Starlight, Dark -Sky Areas and Observatory Sites. In: RUGGLES, C.; COTTE, M. Heritage Sites of Astronomy and Archeoastronomy in the Context of the Unesco World Heritage Convention. Icomos and International Astronomical Union, 2010.

MATOS, A.L. Terrestrial Astrotourism: Motivation and satisfaction of travelling to watch the night sky. 2017. 194 f. Thesis (Tourism Master's Degree Programme), Aalborg University, Aalborg, 2017. Disponível em:

<https://projekter.aau.dk/projekter/files/260343239/THESIS_ASTROTOURISM_PDF. pdf>. Acesso em: 27 abr. 2019. 
MOREIRA, D. A. O método fenomenológico na pesquisa. São Paulo: Pioneira Thomson, 2002.

MORELLE, R. Light pollution 'affects $80 \%$ of global population'. BBC - Science Environment, 2016. Disponível em: <https://www.bbc.com/news/scienceenvironment-36492596>. Acesso em: 20 abr. 2019.

NAJAFABADI, S. S. Astronomical Tourism (Astro-Tourism) in Cebu, Philippines: essential features in selected destinations and its complementing visitor attractions. Bangkok (Thailand). Anais... Bangkok (Thailand): International Conference on Trade, Tourism and Management, v. 21/22, p. 129-133, 2012.

RICH, C.; LONGCORE, T. Ecological consequences of artificial night lighting. Washington, DC: IslandPress, 2013.

RODRIGUES, A. L. O.; RODRIGUES, A.; PEROFF, D. M. The Sky and Sustainable Tourism Development: A Case Study of a Dark Sky Reserve Implementation in Alqueva. International Journal of Tourism Research, v. 17, n. 3, p.292-302, jan. 2014.

SÃO PAUlO. Secretaria de Infraestrutura e Meio Ambiente. Parque do Morro do Diabo é opção de área ecológica no oeste paulista, 2010. Disponível em: < http://www.saopaulo.sp.gov.br/ultimas-noticias/parque-do-morro-do-diabo-e-opcao-dearea-ecologica-no-oeste-paulista/>. Acesso em 21 de abril de 2019.

SÃO PAULO. Secretaria de Infraestrutura e Meio Ambiente. Morro do Diabo já foi parte de um grande deserto, 2017. Disponível em: < https://www.infraestruturameioambiente.sp.gov.br/2017/07/morro-do-diabo-ja-foiparte-de-um-grande-deserto/>. Acesso em 21 de abril de 2019.

SOLÁ, E. F.; CABRERA, C.M.; JAFARI, J. Astrotourism: No Requiem for Meaningful Travel. Pasos Revista de Turismo y Patrimonio Cultural, v. 12, n.4, p.663-671, 2014.

SPENNEMANN, D. H. R. Orbital lunar and interplanetary tourism. In C. Marin \& J. Jafari (Eds.), Starlight: A common heritage, Proceedings of the International Astronomical Union, p. 363-375, 2008.

TOMANIK, G. B.; BASTOS, S. R. Um patrimônio da humanidade ameaçado: o céu noturno. São Paulo. Anais... São Paulo: Seminário da ANPTUR - Turismo e Patrimônio. Aleph, 1, pp. 1-13, 2012.

UNESCO. Proclamation of 2009 as the United Nations International Year of Astronomy. General Conference, Paris, 2009, 6p.

VERGARA, S. C. Projetos e relatórios de pesquisa em administração. 3.ed. Rio de Janeiro: Atlas, 2000.

Recebido em: 20-10-2019

Aprovado em: 03-02-2020 